\title{
The Effect of Big Five Personality Traits on Physics Education in Middle School: Evidence from a Chinese Study
}

\author{
Shui-hua WANG ${ }^{1,2,3}$, Yu-feng JIA ${ }^{2}$, Yi CHEN ${ }^{1}$, Hui-min LU ${ }^{4}$, John LIU ${ }^{5}$, \\ Ming $\mathrm{YANG}^{6}$ and $\mathrm{Yu}$-dong $Z \mathrm{HANG}^{1,3, \mathrm{a},{ }^{*}}$ \\ ${ }^{1}$ School of Computer Science and Technology, Nanjing Normal University, \\ Nanjing, Jiangsu 210023, P R China \\ 2 Jinchang Experimental Middle School, Suzhou, Jiangsu 215008, P R China \\ ${ }^{3}$ Jiangsu Key Laboratory of Advanced Manufacturing Technology, \\ Huaiyin, Jiangsu 223003, China \\ ${ }^{4}$ Department of Mechanical and Control Engineering, Kyushu Institute of Technology, \\ Fukuoka Prefecture 804-8550, Japan \\ ${ }^{5}$ College of Engineering, Michigan State University, East Lansing, MI 48823, USA \\ ${ }^{6}$ Department of Radiology, Nanjing Children's Hospital, Nanjing Medical University, \\ Nanjing 210008, China \\ azhangyudong@njnu.edu.cn
}

Keywords: Big Five personality traits, Physics score, Middle school, Correlation analysis.

\begin{abstract}
To explore the correlation between personality traits and physics score, we enrolled 60 students as samples, and obtained their Big Five personality traits by NEOFFI questionnaire. The average of the scores of the midterm examination and final examination of both Grade 7 and Grade 8 is used as the predictor variable. The correlation analysis showed that the conscientiousness is positively related to physics score, and the neuroticism is negatively related to physics score. Other three personality traits are not related to physics score.
\end{abstract}

\section{Introduction}

The Big Five personality traits (BFPT) measure is an important personality descriptor [1]. These measures contain five factors: openness to experience, conscientiousness, extraversion, agreeableness, and neuroticism. In common, the aforementioned five factors are abbreviated as OCEAN. The BFPT has intimate connections with friendship [2], nonverbal intelligence [3], deviant behavior [4], internet addition [5], etc.

Except emotion and psychology, the scholars also investigated the relationship between Big Five personality dimensions with primary and secondary educations. de Bruin, et al. [6] explored whether scores on intelligence tests and personality questionnaires can predict performance in an adult basic education and training (ABET) program.Rammstedt, et al. [7] demonstrated the measurement equivalence of Big Five factor markers for persons with different level of education. Culjak and Mlacic [8] used Big Five model to predict the success of high school students. Smidt, et al. [9] provided a German study to discover how Big Five personality traits influence the early childhood pedagogues and their beliefs. 
This study is a complementarity of aforementioned studies. Our contribution contains two points: (1) we enrolled Chinese intermediate students as subjects. (2) Our study is for physical education. The rest of this paper is structured as follows: Next Section Ogives the methodology. Section 0 presents the results and discussions. Finally, Section 0 concludes the paper.

\section{Methodology}

\section{Subjects}

We enrolled in total 60 junior middle school students (Grade 8), with their demographics listed in Table 1. There are 29 male students and 31 female students. From the view of age, there are 19 students with age of 13, 24 students with age of 14, 13 students at age of 15 , and 4 students at age of 16 . There are 32 students follows one-child policy. All students live in the city.

Table 1 Demographics of 60 subjects from a junior middle school

\begin{tabular}{ll}
\hline Characteristics & Value \\
\hline Gender & Male (29), Female (31) \\
Age & $13(19), 14(24), 15(13), 16(4)$ \\
One-Child & No (32), Yes (28) \\
Location & City (60), Suburb (0) \\
Grade & Grade 8 (60) \\
\hline The digits in the parenthesis represent the number of people
\end{tabular}

\section{Questionnaire}

The revised NEO personality inventory (NEO PI-R)[10] contains in total 240 items. Two forms exist: self-report and observe report. It costs too much time for the students to finish that questionnaire; hence we used the shortened version, 60-item inventory, named asNEO-Five Factor Inventory (NEO-FFI)[11]. The personality dimension and corresponding facets are listed in Table 2. Note that NEO was considered as NeuroticismExtroversion-Openness Inventory, but now it is no longer an acronym. The scores of this 60-item NEO-FFI were used as the dependent variable.

Table 2 Personality dimension

\begin{tabular}{ll}
\hline Personality dimension & Facets \\
\hline Openness to experience & Fantasy, Aesthetics, Feelings, Actions, Ideas, Values \\
Conscientiousness & Competence, Order, Dutifulness, Achievement Striving, Self-Discipline, \\
& Deliberation \\
Extraversion & Warmth, Gregariousness, Assertiveness, Activity, Excitement Seeking, \\
& Positive Emotion \\
Agreeableness & Trust, Straightforwardness, Altruism, Compliance, Modesty, Tender \\
& mindedness \\
Neuroticism & Anxiety, Hostility, Depression, Self-Consciousness, Impulsiveness, \\
& Vulnerability to Stress \\
\hline
\end{tabular}


We record the scores of the midterm examination and final examination of both Grade 7 and Grade 8 . The averaged physical exam score was used as the predictor variable.

\section{Software}

We used Matlab 2016b as the computing platform. Correlation analysis [12]was used to make statistical test whether Big Five personality dimension is related to physics score. The command "corr", shown in Table 3, was used to carry out the correlation analysis. The command outputs the linear correlation coefficient $(\rho)$ and the p-value $(P)$.

Table 3 Matlab command of "corr"

\begin{tabular}{l}
\hline$[\rho, \mathrm{P}]=\operatorname{corr}(\mathrm{X}, \mathrm{Y})$ \\
\hline Input \\
$\mathrm{X}$ : dependent variable \\
$\mathrm{Y}$ : independent variable \\
\hline Output \\
$\rho$ : linear correlation coefficient \\
$\mathrm{P}: \mathrm{p}$-values
\end{tabular}

The Pearson's linear correlation coefficient was calculated based on the "corr" command. Two-tailed tests are used. The alternate hypothesis is that the correlation is not zero, and the null hypothesis is that the correlation is zero.

\section{Results and Discussions}

Table 4 Correlation analysis of physics score with gender, age, and one-child factors

\begin{tabular}{llll}
\hline & Gender & Age & One-child \\
\hline$\rho$ & -0.010 & -0.2148 & -0.0039 \\
$\mathrm{P}$ & 0.9335 & 0.0993 & 0.9764 \\
\hline
\end{tabular}

Table 5 Correlation analysis of physics score with gender, age, and one-child factors

\begin{tabular}{llllll}
\hline & Openness to experience & Conscientiousness & Extraversion & Agreeableness & Neuroticism \\
\hline$\rho$ & 0.1564 & 0.3941 & 0.0872 & -0.0738 & -0.3582 \\
$\mathrm{P}$ & 0.2327 & $0.0018^{*}$ & 0.5076 & 0.5752 & $0.0050^{*}$ \\
\hline$(* \mathrm{P}<0.05)$ & & &
\end{tabular}

$(* \mathrm{P}<0.05)$

Table 4 shows that the gender, age, and one-child policy do not have significant correlation with physics scores. Table 5 shows that the conscientiousness is positively correlated with physics score $(\rho=0.3941, \mathrm{P}=0.0018)$. This result can help our teachers in cultivating students in learning physics. Except the contents in the textbook, the teachers should cultivate students in the characteristics of self-discipline and striving for achievement. Besides, the students should learn control and regulate themselves. Spontaneous behaviors should be avoided.

Table 5 also shows that the neuroticism is negatively correlated with physics score $(\rho=$ $-0.3582, \mathrm{P}=0.0050)$. The result indicates that calm and emotional stability can benefit physics learning. The students should be far away from negative emotions.

The questionnaire is somewhat subject, and may be influenced by personal feelings. We shall try to use neuroimaging techniques, e.g., the magnetic resonance imaging [1318], to scan the brain to obtain its structure and functioning states. More advances statistical techniques, computer vision methods [19, 20], and artificial intelligence approaches [21, 22] shall be tested. 


\section{Conclusions}

In this study, we find the conscientiousness is positively related to physics score, and the neuroticism is negatively related to physics score. Other three personality traits are not related to physics score.

In the future, we shall try to use the NEO PI-R questionnaire. We shall also collect the scores of the students on mathematics, English, chemistry, history, etc. We shall use correlation analysis to know their relationship with Big Five personality traits.

\section{Acknowledgement}

This study was supported by Natural Science Foundation of China (61602250), Leading Initiative for Excellent Young Researcher (LEADER) of Ministry of Education, Culture, Sports, Science and Technology-Japan (16809746), Natural Science Foundation of Jiangsu Province (BK20150983), Program of Natural Science Research of Jiangsu Higher Education Institutions (16KJB520025, 15KJB470010), Open Program of Jiangsu Key Laboratory of 3D Printing Equipment and Manufacturing (3DL201602), Open fund for Jiangsu Key Laboratory of Advanced Manufacturing Technology (HGAMTL1601)

\section{References}

[1] H. Heidemeier and A. S. Goritz, "The Instrumental Role of Personality Traits: Using Mixture Structural Equation Modeling to Investigate Individual Differences in the Relationships Between the Big Five Traits and Life Satisfaction," Journal of Happiness Studies, vol. 17, Dec 2016 pp. 2595-2612,

[2] K. Harris and S. Vazire, "On friendship development and the Big Five personality traits," Social And Personality Psychology Compass, vol. 10, Nov 2016 pp. 647-667,

[3] I. Voronina, A. Fenin, T. Malykh, and A. Belova, "Relationships of Big Five personality traits and nonverbal intelligence at high school age," in International Conference Education Environment for the Information Age, Moscow, RUSSIA, 2016.

[4] A. Abdullah and S. Marican, "The Effects of Big-Five Personality Traits on Deviant Behavior," in 3rd Global Conference on Business And Social Sciences, Dubai, U ARAB EMIRATES, 2016, pp. 19-25.

[5] A. R. Kayis, S. A. Satici, M. F. Yilmaz, D. Simsek, E. Ceyhan, and F. Bakioglu, "Big five-personality trait and internet addiction: A meta-analytic review," Computers In Human Behavior, vol. 63, Oct 2016 pp. 35-40,

[6] K. de Bruin, G. P. de Bruin, S. Dercksen, and M. Cilliers-Hartslief, "Predictive validity of general intelligence and Big Five measures for adult basic education and training outcomes," South African Journal Of Psychology, vol. 35, Mar 2005 pp. 46-57,

[7] B. Rammstedt, L. R. Goldberg, and I. Borg, "The measurement equivalence of BigFive factor markers for persons with different levels of education," Journal Of Research In Personality, vol. 44, Feb 2010 pp. 53-61, 
[8] Z. Culjak and B. Mlacic, "The Big-Five Model of Personality and the Success of High School Students in Physical Education," Croatian Journal Of Education, vol. 16, 2014 pp. 471-490,

[9] W. Smidt, G. Kammermeyer, and S. Roux, "Relations between the Big Five personality traits of prospective early childhood pedagogues and their beliefs about the education of preschool children: Evidence from a German study," Learning And Individual Differences, vol. 37, Jan 2015 pp. 96-106,

[10] B. Calvet, M. Bricaud, and J. P. Clement, "Relationship between two personality assessment inventories (TCI and NEO PI-R) in old individuals," International Psychogeriatrics, vol. 27, Dec 2015 pp. S146-S146,

[11] J. Henriques-Calado, M. E. Duarte-Silva, and A. S. Ferreira, "Personality traits in women with Alzheimer's disease: Comparisons with control groups with the NEO-FFI," Personality And Individual Differences, vol. 101, Oct 2016 pp. 341-347,

[12] A. T. McKenzie, I. Katsyv, W. M. Song, M. H. Wang, and B. Zhang, "DGCA: A comprehensive R package for Differential Gene Correlation Analysis," BMC Systems Biology, vol. 10, Nov 2016 p. 25, Article ID: 106

[13]Z. Dong, "Classification of Alzheimer disease based on structural magnetic resonance imaging by kernel support vector machine decision tree," Progress In Electromagnetics Research, vol. 144, 2014 pp. 171-184,

[14]D. Yu, H. Shui, L. Gen, and C. Zheng, "Exponential wavelet iterative shrinkage thresholding algorithm with random shift for compressed sensing magnetic resonance imaging," IEEJ Transactions on Electrical and Electronic Engineering, vol. 10, 2015 pp. 116-117,

[15]Z. Dong, P. Phillips, G. Ji, and J. Yang, "Exponential wavelet iterative shrinkage thresholding algorithm for compressed sensing magnetic resonance imaging," Information Sciences, vol. 322, 11/20/ 2015 pp. 115-132,

[16]P. Phillips, Z. Dong, and J. Yang, "Pathological brain detection in magnetic resonance imaging scanning by wavelet entropy and hybridization of biogeography-based optimization and particle swarm optimization," Progress In Electromagnetics Research, vol. 152,2015 pp. 41-58,

[17] G. Liu, P. Phillips, and T.-F. Yuan, "Detection of Alzheimer's Disease by ThreeDimensional Displacement Field Estimation in Structural Magnetic Resonance Imaging," Journal of Alzheimer's Disease, vol. 50, 2016 pp. 233-248,

[18]T. Zhan, "Pathological brain detection by artificial intelligence in magnetic resonance imaging scanning," Progress in Electromagnetics Research, vol. 156, 2016 pp. 105-133,

[19] L. Wu, "Classification of fruits using computer vision and a multiclass support vector machine," Sensors, vol. 12, 2012 pp. 12489-12505,

[20] G. Ji, "Fruit classification using computer vision and feedforward neural network," Journal of Food Engineering, vol. 143, 2014 pp. 167-177, 
[21] S. Balochian, "Artificial Intelligence and Its Applications," Mathematical Problems in Engineering, 2014 Article ID: 840491

[22]P. Agarwal, V. Bhatnagar, and S. Balochian, "Swarm Intelligence and Its Applications," The Scientific World Journal, 2013 Article ID: 528069 